\title{
Thymus praecox subsp. skorpilii var. skorpilii extract attenuates degeneration of beta-cells and reduced insulin levels in type 2 diabetic rats
}

\section{Ayse Nur Hazar-Yavuz ${ }^{1}$, Muhammet Emin Cam ${ }^{1}$, Sila Yildiz1, Turgut Taskin², Saadet Alan, Levent Kabasakal ${ }^{1}$}

${ }^{I}$ Pharmacology, Faculty of Pharmacy, Marmara University, Turkey, ${ }^{2}$ Pharmacognosy, Faculty of Pharmacy, Marmara University, Turkey, ${ }^{3}$ Pathology, Faculty of Medicine, Inonu University, Turkey

Background

T2DM is characterized by hyperglycemia that body cells inadequately response to insulin and/or pancreatic beta-cells are partly impaired. Thymus praecox subsp. skorpilii var. skorpilii (TPS) leaves are traditionally used for the treatment of diabetes in Turkey. In our previous study, we firstly evaluated the antidiabetic effect of TPS extract (TPSE). Hence we designed this study to uncover the mechanism of action of TPSE in T2DM induced by streptozotocin/nicotinamide (STZ/NA) in rats.

Methods

All experimental protocols were approved by the Marmara University, Animal Experiments Local Ethics Committee (protocol number:098.2016.mar). Diabetes was induced with STZ/NA injection (i.p.) in Sprague-Dawley rats. Animals were randomly divided into 4 groups $(n=6)$ : Control Group, Diabetes Group, TPSE Group, Positive Control Group; rats were administered $10 \mathrm{ml} / \mathrm{kg}$ saline (first two groups), $100 \mathrm{mg} / \mathrm{kg}$ TPSE, $400 \mathrm{mg} / \mathrm{kg}$ metformin by intragastic gavage, respectively. TPSE and metformin treatments were started 3 days after the induction of diabetes and continued for 3 weeks. At the end of 3 weeks, all rats were decapitated and serum samples were collected for ELISA analyzes. The pancreas tissues were fixed in $10 \%$ buffered formalin for histopathologic analyzes. Statistical analysis was performed by GraphPad Prism 6.0 software. Data of groups were compared with one-way analysis of variance (ANOVA) and Tukey post-hoc test. Values were considered significantly different. All data were expressed as standard error of mean.

Results

In progressive stages of T2DM, serum insulin levels seriously decrease due to destruction of beta-cells in rats. Insulin levels were dropped dramatically in untreated diabetic rats compared to healthy rats. Moreover, insulin levels of treatment groups almost reached to those of control group. While the degeneration of beta-cells was observed in diabetic rats, control rats showed normal cellular population. TPSE treatment resulted in a significant increase in small beta cells. This improvement correlates increased level of insulin in TPSE treated rats compared to untreated diabetic rats.

Conclusions

We therefore conclude that some of the mechanisms of action of Thymus praecox subsp. skorpilii var. skorpilii in T2DM are enhancing degeneration of beta-cells and reduced insulin levels. 